\title{
Differential Flatness Properties and Feedback Control of the Fokker-Planck PDE
}

\author{
G. Rigatos ${ }^{1} \cdot$ P. Siano ${ }^{2} \cdot$ A. Melkikh ${ }^{3}$
}

Received: 3 August 2016/ Accepted: 9 August 2016 / Published online: 24 September 2016

(C) Springer Science+Business Media Singapore 2016

\begin{abstract}
The Fokker-Planck PDE describes the dynamics of several biochemical and biological processes, as well as of phenomena at molecular scale. In this paper, the problem of feedback control of the Fokker-Planck PDE is studied. It is shown that the procedure for numerical solution of Fokker-Planck PDE results into a set of nonlinear ordinary differential equations (ODEs) and an associated state equations model. For the local subsystems, into which a Fokker-Planck PDE is decomposed, it becomes possible to apply boundary-based feedback control. The controller design proceeds by showing that the state-space model of the Fokker-Planck PDE stands for a differentially flat system. Next, for each subsystem which is related to a nonlinear ODE, a virtual control input is computed, that can invert the subsystem's dynamics and can eliminate the subsystem's tracking error. From the last row of the state-space description, the control input (boundary condition) that is actually applied to the Fokker-Planck PDE system is found. This control input contains recursively all virtual control inputs which were computed for the individual ODE subsystems associated with the previous rows of the state-space equation. Thus, by tracing the rows of the state-space model backwards, at
\end{abstract}

\section{G. Rigatos}

grigat@ieee.org

P. Siano

psiano@unisa.it

A. Melkikh

melkikh2008@ rambler.ru

1 Unit of Industrial Automation, Industrial Systems Institute, 26504 Rion Patras, Greece

2 Department of Industrial Engineering, University of Salerno, 84084 Fisciano, Italy

3 Institute of Physics and Technology, Ural Federal University, Yekaterinburg, Russia 620002 each iteration of the control algorithm, one can finally obtain the control input that should be applied to the Fokker-Planck PDE system so as to assure that all its state variables will converge to the desirable setpoints.

Keywords Fokker-Planck PDE - Diffusion process . Nonlinear control · PDE boundary control · Asymptotic stability $\cdot$ Lyapunov analysis

\section{Introduction}

The Fokker-Planck PDE is a parabolic-type partial differential equation which describes the diffusive motion of a particle under an external potential. Under specific conditions the Fokker-Planck PDE is shown to be equivalent to Langevin's (and to the Ornstein-Uhlenbeck) stochastic differential equation [1-4]. In the latter case, the diffusing particle is subject to the following forces: (i) a spring force (drift) which is the result of the harmonic potential and tries to drive the particle to an equilibrium and (ii) a random force (noise) which is the result of the interaction with neighboring particles [5-7]. The Fokker-Planck PDE defines the dynamics of several biochemical and biological processes, as well as of phenomena at molecular scale [8-11]. This PDE, also known as Kolmogorov processive equation shows the spatiotemporal variation of a probability density function $\rho(x, t)$ which gives the position of the particle at location $x$ and time instant $t$. Usually solutions of this PDE are computed for specific types of external potential. In other cases a stationary solution of the PDE is assumed considering that the probability density function does not vary in time and thus $\rho(x, t)=\rho_{0}(x)$. The control problem of the Fokker-Planck $\mathrm{PDE}$ is a non trivial one. 
The present paper applied semi-discretization for the numerical representation of the PDE dynamics, which is an approach also implemented in [12-16]. Moreover, this paper treats the problem of boundary control of the nonlinear Fokker-Planck PDE, which means that the boundary conditions are used as control inputs to modify this PDE dynamics. By decomposing the PDE system into an equivalent set of ordinary nonlinear differential equations, then controlling such a set of dynamical subsystems by the boundary conditions implies underactuation (actually one has to control the different degrees of freedom associated with the set of nonlinear ODEs by using only a couple of control inputs).

Methods for feedback stabilization of systems with nonlinear PDE dynamics have been a flourishing research subject in the last years [17-20]. In particular, feedback control of diffusion-type (parabolic) PDEs has been a subject of extensive research and several remarkable results have been produced [21-24]. For the control of the heat diffusion PDE boundary and distributed control methods have been developed [25-30]. The control approach followed in this paper is as follows. First, by implementing a finite differences procedure a set of equivalent nonlinear ordinary differential equations (ODEs) is obtained for the Fokker-Planck PDE. Next it is shown that the system of the nonlinear ODEs is a differentially flat one. This means that all its state variables and the control inputs can be written as differential functions of one single algebraic variable which is the flat output [31-36].

Actually, by examining independently each nonlinear ODE it is shown that this stands again for a differentially flat system, for which a virtual control input can be computed as in the case of flatness-based control for the trivial system. The virtual control input is chosen such that the ODE subsystem dynamics is linearized and the tracking error is eliminated. The boundary condition that appears in the nonlinear ODE subsystem and which comprises the last rows of the statespace description, stands for the aggregate control input. The computation of the boundary control input uses recursively all virtual control inputs mentioned above, moving from the last ODE system to the first one [35]. Thus, by tracing the rows of the state-space model backwards, at each iteration of the control algorithm, one can finally obtain the control input that should be applied to the Fokker-Planck PDE system so as to assure that all its state vector elements will converge to the desirable setpoints. By analyzing the dynamics of the closed loop system that results from the application of the aforementioned control method, asymptotic stability is confirmed.

The structure of the paper is as follows: in "Transformation of the Fokker-Planck PDE into a Set of Nonlinear ODEs" section the state-space description of the Fokker-Planck PDE is analyzed. In "Differential Flatness of the Fokker-Planck PDE Model" section the differential flatness properties of the Fokker-Planck PDE are confirmed. In "Computation of a Boundary Conditions-Based Feedback Control Law" section a boundary conditions-based feedback control law is computed for the Fokker-Planck PDE. In "Closed Loop Dynamics" section the dynamics of the closed control loop of the Fokker-Planck PDE is analyzed and stability proof is given. In "Simulation Tests" section simulation tests are provided to evaluate the performance of the proposed PDE control method. Finally, in "Conclusions" section concluding remarks are stated.

\section{Transformation of the Fokker-Planck PDE into a Set of Nonlinear ODEs}

\section{The Fokker-Planck PDE}

The Fokker-Planck PDE describes the dynamics of several biochemical and biological processes, as well as of phenomena at molecular scale. The Fokker-Planck PDE is a parabolic-type partial differential equation which describes the diffusive motion of a particle under an external potential. Under specific conditions the Fokker-Planck PDE is shown to be equivalent to Langevin's (and to the OrnsteinUhlenbeck) stochastic differential equation. In the latter case, the diffusing particle is subject to the following forces: (i) a spring force (drift) which is the result of the harmonic potential and tries to drive the particle to an equilibrium and (ii) a random force (noise) which is the result of the interaction with neighboring particles.

It is assumed that $\rho(x, t)$ denotes the probability density function for the particle's position to take the value $x$ at time instant $t$. One can obtain the Fokker-Planck equation after a series of transformations applied to Langevin's equation, thus confirming that the two relations are equivalent. Thus one starts from a SDE of the form

$d x=a(x, t) d t+b(x, t) d w$

Next the transformation $y=h(x)$ is introduced, where $h$ is an arbitrary function that is twice differentiable. It holds that

$d h(x)=h^{\prime}(x) a(x, t) d t+h^{\prime \prime} b^{2}(x, t) / 2 d t+h^{\prime}(x) b(x, t) d w$

By computing mean values one gets

$\frac{d}{d t} E\{h(x)\}=E\left\{h^{\prime}(x) a(x, t)\right\}+E\left\{h^{\prime \prime}(x) b^{2}(x, t) / 2\right\}$

Assume that $\rho(x, t)$ is the probability density function for the stochastic variable $x$. It is noted that

$E\{U(x, t)\}=\int U(x, t) \rho(x, t) d x$ 
Using Taylor series expansion one gets

$$
\begin{aligned}
\frac{d}{d t} \int h(x) \rho(x, t) d x= & \int\left[h^{\prime}(x) a(x, t)\right. \\
& \left.+h^{\prime \prime} b^{2}(x, t) / 2\right] \rho(x, t) d x
\end{aligned}
$$

which is also written as

$$
\begin{aligned}
\frac{d}{d t} \int h(x) \rho(x, t) d x= & \int\left[-(a(x, t) \rho(x, t))^{\prime}\right. \\
& \left.+\left(b^{2}(x, t) / 2 \rho(x, t)\right)^{\prime \prime}\right] h(x) d x
\end{aligned}
$$

and since the relation described in Eq. (6) should hold for all $h(x)$ one gets

$\frac{\partial \rho}{\partial t}=\frac{\partial}{\partial x}\left[-a(x, t) \rho(x, t)+\frac{1}{2} \frac{\partial}{\partial x}\left[b^{2}(x, t) \rho(x, t)\right]\right]$

This is Fokker-Planck equation.

\section{Modeling in State-Space Form of the Fokker-Planck PDE}

In the previously given Fokker-Planck PDE of Eq. (7), it is considered that $a(x, t)=-\alpha x$ and $b(x, t)=b$. Therefore, one arrives at the following form of the Fokker-Planck PDE

$\frac{\partial \rho}{\partial t}=\frac{1}{2} b^{2} \frac{\partial^{2} \rho}{\partial x^{2}}+\frac{\partial}{\partial x}[\alpha x \rho]$

which in turn is written as

$\frac{\partial \rho}{\partial t}=\frac{1}{2} b^{2} \frac{\partial^{2} \rho}{\partial x^{2}}+\alpha P+\alpha x \frac{\partial}{\partial x} \rho$

which can be also written in the form

$\frac{\partial \rho}{\partial t}=\frac{1}{2} b^{2} \nabla^{2} \rho+\alpha \rho+\alpha x \nabla \rho$

A grid of $N$ points is considered, that is $\left\{x_{1}, x_{2}, \ldots, x_{N-1}\right.$, $\left.x_{N}\right\}$ which are placed at equal distances on the $x$ axis. At the points of spatial discretization it holds

$\frac{\partial \rho_{i}}{\partial t}=\frac{1}{2} b^{2} \frac{\rho_{i+1}-2 \rho_{i}+\rho_{i-1}}{\Delta x^{2}}+\alpha \rho_{i}+\alpha x_{i} \frac{\rho_{i+1}-\rho_{i}}{\Delta x}$

where $\rho_{0}=\rho\left(x_{0}, t\right)$ and $\rho_{N+1}=\rho\left(x_{N+1}, t\right)$ are taken to be boundary conditions. For the $i$-th ODE one has at sampling point $x_{i}$ along the $x$ axis one has

$$
\begin{aligned}
\frac{\partial \rho_{i}}{\partial t}= & \frac{1}{2} b^{2} \frac{\rho_{i-1}}{\Delta x^{2}}-\frac{1}{2} b^{2} \frac{2 \rho_{i}}{\Delta x^{2}}+\frac{1}{2} b^{2} \frac{\rho_{i+1}}{\Delta x^{2}} \\
& +\alpha \rho_{i}+\alpha x_{i} \Delta x \frac{\rho_{i+1}-\rho_{i}}{\Delta x^{2}}
\end{aligned}
$$

Equation (12) is also written in the form

$$
\begin{aligned}
\frac{\partial \rho_{i}}{\partial t}= & \frac{1}{2} b^{2} \frac{\rho_{i-1}}{\Delta x^{2}}-\frac{1}{2} b^{2} \frac{2 \rho_{i}}{\Delta x^{2}}+\left[\frac{1}{2} b^{2}+\alpha x_{i} \Delta x\right] \frac{\rho_{i+1}}{\Delta x^{2}} \\
& +\alpha \rho_{i}-\alpha x_{i} \Delta x \frac{\rho_{i}}{\Delta x^{2}}
\end{aligned}
$$

By denoting $K_{1}=\frac{1}{2} b^{2}, K_{2}=\frac{1}{2} b^{2}+\alpha x_{i} \Delta x$ and $f\left(\rho_{i}\right)=$ $\alpha \rho_{i}-\alpha x_{i} \Delta x \frac{\rho_{i}}{\Delta x^{2}}$ one obtains the following description for Eq. (13)

$\frac{\partial \rho_{i}}{\partial t}=\frac{K_{1}}{\Delta x^{2}} \rho_{i-1}-\frac{2 K_{1}}{\Delta x^{2}} \rho_{i}+\frac{K_{2}}{\Delta x^{2}} \rho_{i+1}+f\left(\rho_{i}\right)$

\section{Differential Flatness of the Fokker-Planck PDE Model}

Next, the following state vector is defined for the PDE model: $\tilde{Y}=\left[y_{1,1}, y_{1,2}, \ldots, y_{1, i}, \ldots, y_{1, N-1}, y_{1, N}\right]$, where $y_{1,1}=$ $\rho_{1}, y_{1,2}=\rho_{2}, \ldots, y_{1, i}=\rho_{i}, \ldots, y_{1, N-1}=\rho_{N-1}$ and $y_{1, N}=$ $\rho_{N}$. It will be shown that the state-space description of the nonlinear PDE dynamics, which has as control input only the boundary condition $\rho_{0}$ is differentially flat. One has

$$
\begin{aligned}
\dot{y}_{1,1}= & -\frac{2 K_{1}}{\Delta x^{2}} y_{1,1}+\frac{K_{1}}{\Delta x^{2}} \rho_{0}+f\left(y_{1,1}\right)+\frac{K_{2}}{\Delta x^{2}} y_{1,2} \\
\dot{y}_{1,2}= & -\frac{2 K_{1}}{\Delta x^{2}} y_{1,2}+\frac{K_{1}}{\Delta x^{2}} y_{1,1}+f\left(y_{1,2}\right)+\frac{K_{2}}{\Delta x^{2}} y_{1,3} \\
\dot{y}_{1,3}= & -\frac{2 K_{1}}{\Delta x^{2}} y_{1,3}+\frac{K_{1}}{\Delta x^{2}} y_{1,2}+f\left(y_{1,3}\right)+\frac{K_{2}}{\Delta x^{2}} y_{1,4} \\
\dot{y}_{1, i}= & -\frac{2 K_{1}}{\Delta x^{2}} y_{1, i}+\frac{K_{1}}{\Delta x^{2}} y_{1, i-1}+f\left(y_{1, i}\right)+\frac{K_{2}}{\Delta x^{2}} y_{1, i+1} \\
\dot{y}_{1, N-1}= & -\frac{2 K_{1}}{\Delta x^{2}} y_{1, N-1}+\frac{K_{1}}{\Delta x^{2}} y_{1, N-2}+f\left(y_{1, N-1}\right) \\
& +\frac{K_{2}}{\Delta x^{2}} y_{1, N} \\
\dot{y}_{1, N}= & -\frac{2 K_{1}}{\Delta x^{2}} y_{1, N}+\frac{K_{1}}{\Delta x^{2}} y_{1, N-1}+f\left(y_{1, N}\right) \\
& +\frac{K_{2}}{\Delta x^{2}} \rho_{N+1}
\end{aligned}
$$

The flat output is considered to be the state variable $y_{1, N}$, which is denoted as $y=y_{1, N}$. Next, it is shown that all state variables which stand also for virtual control inputs of the system $\alpha_{i}=y_{1, i+1}$, can be written as functions of the flat 
output $y=y_{1, N}$. From Eq. (20) and considering that $\rho_{N+1}$ is constant one obtains

$$
\begin{aligned}
y_{1, N-1}= & \alpha_{1}=\frac{1}{K_{1} / \Delta x^{2}}\left[\dot{y}_{1, N}+\frac{2 K_{1}}{\Delta x^{2}} y_{1, N}-\frac{K_{2}}{\Delta x^{2}} \rho_{N+1}\right. \\
& \left.-f\left(y_{1, N}\right)\right] \Rightarrow y_{1, N-1}=h_{1}(y, \dot{y}, \ldots)
\end{aligned}
$$

and following a similar procedure, from Eq. (19) one gets

$$
\begin{aligned}
y_{1, N-2}= & \alpha_{2}=\frac{1}{K_{1} / \Delta x^{2}} \\
& \times\left[\dot{y}_{1, N-1}+\frac{2 K_{1}}{\Delta x^{2}} y_{1, N-1}-\frac{K_{2}}{\Delta x^{2}} y_{1, N}\right. \\
& \left.-f\left(y_{1, N-1}\right)\right] \Rightarrow y_{1, N-2}=h_{2}(y, \dot{y}, \ldots)
\end{aligned}
$$

Continuing in a similar manner, from Eq. (18) one obtains

$$
\begin{aligned}
y_{1, i-1}= & \alpha_{N-i+1}=\frac{1}{K_{1} / \Delta x^{2}} \\
& \times\left[\dot{y}_{1, i}+\frac{2 K_{1}}{\Delta x^{2}} y_{1, i}-\frac{K_{2}}{\Delta x^{2}} y_{1, i+1}\right. \\
& \left.-f\left(y_{1, i}\right)\right] \Rightarrow y_{1, i-1}=h_{N-i+1}(y, \dot{y}, \ldots)
\end{aligned}
$$

From Eq. (17) one obtains

$$
\begin{aligned}
y_{1,2}= & \alpha_{N-2}=\frac{1}{K_{1} / \Delta x^{2}}\left[\dot{y}_{1,3}+\frac{2 K_{1}}{\Delta x^{2}} y_{1,3}-\frac{K_{2}}{\Delta x^{2}} y_{1,4}\right. \\
& \left.-f\left(y_{1,3}\right)\right] \Rightarrow y_{1,2}=h_{N-2}(y, \dot{y}, \ldots)
\end{aligned}
$$

From Eq. (16) one obtains

$$
\begin{aligned}
y_{1,1}= & \alpha_{N-1}=\frac{1}{K_{1} / \Delta x^{2}}\left[\dot{y}_{1,2}+\frac{2 K_{1}}{\Delta x^{2}} y_{1,2}-\frac{K_{2}}{\Delta x^{2}} y_{1,3}\right. \\
& \left.-f\left(y_{1,2}\right)\right] \Rightarrow y_{1,1}=h_{N-1}(y, \dot{y}, \ldots)
\end{aligned}
$$

Finally, From Eq. (15) one obtains

$$
\begin{aligned}
\rho_{0}= & \alpha_{N}=\frac{1}{K_{1} / \Delta x^{2}}\left[\dot{y}_{1,1}+\frac{2 K_{1}}{\Delta x^{2}} y_{1,1}-\frac{K_{2}}{\Delta x^{2}} y_{1,2}\right. \\
& \left.-f\left(y_{1,1}\right)\right] \Rightarrow \rho_{0}=h_{N}(y, \dot{y}, \ldots)
\end{aligned}
$$

The above procedure confirms that all state variables of the model

$y_{1,1} y_{1,2} y_{1,3} \ldots y_{1, i} \ldots y_{1, N-1} y_{1, N}$

and the control input which is the boundary condition $\rho_{0}$ can be written as functions of the flat output $y=y_{1, N}$ and of the flat output's derivatives. Consequently, the state-space model of the PDE dynamics is differentially flat.
Additionally, one can consider decomposition of the PDE state-space equation into submodels, where at each submodel the virtual control input is $\alpha_{i}=y_{1, i+1}$

$\dot{y}_{1, i}=-\frac{2 K_{1}}{\Delta x^{2}} y_{1, i}+\frac{K_{1}}{\Delta x^{2}} y_{1, i-1}+f\left(y_{1, i}\right)+\frac{K_{2}}{\Delta x^{2}} y_{1, i+1}$

and with local flat output $y_{1, i}$ one can confirm that all such subsystems $i=1,2, \ldots, N$ are differentially flat. Next, one can compute the virtual inputs which should be applied to each subsystem.

\section{Computation of a Boundary Conditions-Based Feed- back Control Law}

To implement boundary feedback control in the system of Eqs. (15), (20), the nonlinear diffusion-PDE model is rewritten as follows:

$$
\begin{aligned}
\dot{y}_{1, N}= & -\frac{2 K_{1}}{\Delta x^{2}} y_{1, N}+f\left(y_{1, N}\right) \\
& +\frac{K_{2}}{\Delta x^{2}} \rho_{N+1}+\frac{K_{1}}{\Delta x^{2}} y_{1, N-1}
\end{aligned}
$$

$\dot{y}_{1, N-1}=-\frac{2 K_{1}}{\Delta x^{2}} y_{1, N-1}+f\left(y_{1, N-1}\right)+\frac{K_{2}}{\Delta x^{2}} y_{1, N}$

$$
+\frac{K_{1}}{\Delta x^{2}} y_{1, N-2}
$$

$\dot{y}_{1, N-2}=-\frac{2 K_{1}}{\Delta x^{2}} y_{1, N-2}+f\left(y_{1, N-2}\right)+\frac{K_{2}}{\Delta x^{2}} y_{1, N-1}$

$$
\begin{aligned}
& +\frac{K_{1}}{\Delta x^{2}} y_{1, N-1} \\
& \cdots \cdots \\
\dot{y}_{1, i}= & -\frac{2 K_{1}}{\Delta x^{2}} y_{1, i}+f\left(y_{1, i}\right)+\frac{K_{2}}{\Delta x^{2}} y_{1, i+1}+\frac{K_{1}}{\Delta x^{2}} y_{1, i-1} \\
& \cdots \cdots \\
\dot{y}_{1,3}= & -\frac{2 K_{1}}{\Delta x^{2}} y_{1,3}+f\left(y_{1,3}\right)+\frac{K_{2}}{\Delta x^{2}} y_{1,4}+\frac{K_{1}}{\Delta x^{2}} y_{1,2} \\
& \cdots \cdots \\
\dot{y}_{1,2}= & -\frac{2 K_{1}}{\Delta x^{2}} y_{1,2}+f\left(y_{1,2}\right)+\frac{K_{2}}{\Delta x^{2}} y_{1,3}+\frac{K_{1}}{\Delta x^{2}} y_{1,1} \\
\dot{y}_{1,1}= & -\frac{2 K_{1}}{\Delta x^{2}} y_{1,1}+f\left(y_{1,1}\right)+\frac{K_{2}}{\Delta x^{2}} y_{1,2}+\frac{K_{1}}{\Delta x^{2}} \rho_{0}
\end{aligned}
$$

The boundary condition $\rho_{N+1}$ is assumed to be known and remains steady. The boundary condition $\rho_{0}$ stands for the control input. The feedback control law is designed as follows: 


$$
\begin{aligned}
\alpha_{1}= & y_{1, N-1}^{*}=\frac{1}{\left(K_{1} / \Delta x^{2}\right)}\left[\dot{y}_{1, N}^{d}-k_{p, 1}\left(y_{1, N}-y_{1, N}^{d}\right)\right. \\
& \left.+\frac{2 K_{1}}{\Delta x^{2}} y_{1, N}-f\left(y_{1, N}\right)-\frac{K_{2}}{\Delta x^{2}} \rho_{N+1}\right] \\
\alpha_{2}= & y_{1, N-2}^{*}=\frac{1}{\left(K_{1} / \Delta x^{2}\right)}\left[\dot{y}_{1, N-1}^{d}\right. \\
& -k_{p, 2}\left(y_{1, N-1}-y_{1, N-1}^{d}\right)+\frac{2 K_{1}}{\Delta x^{2}} y_{1, N-1} \\
& \left.-f\left(y_{1, N-1}\right)-\frac{K_{2}}{\Delta x^{2}} y_{N}\right] \Rightarrow \\
\alpha_{2}= & \frac{1}{\left(K_{1} / \Delta x^{2}\right)}\left[\dot{\alpha}_{1}-k_{p, 2}\left(y_{1, N-1}-\alpha_{1}\right)\right. \\
& \left.+\frac{2 K_{1}}{\Delta x^{2}} y_{1, N-1}-f\left(y_{1, N-1}\right)-\frac{K_{2}}{\Delta x^{2}} y_{N}\right] \\
\alpha_{3}= & y_{1, N-3}^{*}=\frac{1}{\left(K_{1} / \Delta x^{2}\right)} \\
& \times\left[\dot{y}_{1, N-2}^{d}-k_{p, 3}\left(y_{1, N-2}-y_{1, N-2}^{d}\right)\right. \\
\alpha_{3}= & \frac{2 K_{1}}{\left(K_{1} / \Delta x^{2}\right)}\left[\dot{\alpha}_{2}-k_{p, 3}\left(y_{1, N-2}-\alpha_{2}\right)\right. \\
& \left.+\frac{2 K_{1}}{\Delta x^{2}} y_{1, N-2}-f\left(y_{1, N-2}\right)-\frac{K_{2}}{\Delta x^{2}} y_{N-1}\right] \\
& \left.+\frac{K_{2}}{\Delta x^{2}} y_{N-1}\right] \Rightarrow
\end{aligned}
$$

and continuing in a similar manner

$$
\begin{aligned}
\alpha_{i}= & y_{1, N-i}^{*}=\frac{1}{\left(K_{1} / \Delta x^{2}\right)}\left[\dot{y}_{1, N-i+1}^{d}\right. \\
& -k_{p, i}\left(y_{1, N-i+1}-y_{1, N-i+1}^{d}\right)+\frac{2 K_{1}}{\Delta x^{2}} \\
& \left.y_{1, N-i+1}-f\left(y_{1, N-i+1}\right)-\frac{K_{2}}{\Delta x^{2}} y_{N-i+2}\right] \\
\Rightarrow & \alpha_{i}=\frac{1}{\left(K_{1} / \Delta x^{2}\right)} \\
& \times\left[\dot{\alpha}_{i-1}-k_{p, i}\left(y_{1, N-i+1}-\alpha_{i-1}\right)+\frac{2 K_{1}}{\Delta x^{2}}\right. \\
& \left.y_{1, N-i+1}-f\left(y_{1, N-i+1}\right)-\frac{K_{2}}{\Delta x^{2}} y_{N-i+2}\right]
\end{aligned}
$$

Following this procedure one arrives to compute the control inputs which are associated with the last two rows of the state-space model

$$
\begin{aligned}
\alpha_{N-2}= & y_{1,2}^{*}=\frac{1}{\left(K_{1} / \Delta x^{2}\right)}\left[\dot{y}_{1,3}^{d}-k_{p, N-1}\left(y_{1,3}-y_{1,3}^{d}\right)\right. \\
& \left.+\frac{2 K_{1}}{\Delta x^{2}} y_{1,3}-f\left(y_{1,3}\right)-\frac{K_{2}}{\Delta x^{2}} y_{1,4}\right] \Rightarrow
\end{aligned}
$$

$$
\begin{aligned}
\alpha_{N-2}= & \frac{1}{\left(K_{1} / \Delta x^{2}\right)}\left[\dot{\alpha}_{N-3}-k_{p, N-2}\left(y_{1,3}-\alpha_{N-3}\right)\right. \\
& \left.+\frac{2 K_{1}}{\Delta x^{2}} y_{1,3}-f\left(y_{1,3}\right)-\frac{K_{2}}{\Delta x^{2}} y_{1,4}\right] \\
\alpha_{N-1}= & y_{1,1}^{*}=\frac{1}{\left(K_{1} / \Delta x^{2}\right)}\left[\dot{y}_{1,2}^{d}-k_{p, N-1}\left(y_{1,2}-y_{1,2}^{d}\right)\right. \\
& \left.+\frac{2 K_{1}}{\Delta x^{2}} y_{1,2}-f\left(y_{1,2}\right)-\frac{K_{2}}{\Delta x^{2}} y_{1,3}\right] \Rightarrow \\
\alpha_{N-1}= & \frac{1}{\left(K_{1} / \Delta x^{2}\right)}\left[\dot{\alpha}_{N-2}-k_{p, N-1}\left(y_{1,2}-\alpha_{N-2}\right)\right. \\
& \left.+\frac{2 K_{1}}{\Delta x^{2}} y_{1,2}-f\left(y_{1,2}\right)-\frac{K_{2}}{\Delta x^{2}} y_{1,3}\right]
\end{aligned}
$$

and finally

$$
\begin{aligned}
\alpha_{N}= & \phi_{0}=\frac{1}{\left(K_{1} / \Delta x^{2}\right)}\left[\dot{y}_{1,1}^{d}-k_{p, N}\left(y_{1,1}-y_{1,1}^{d}\right)\right. \\
& \left.+\frac{2 K_{1}}{\Delta x^{2}} y_{1,1}-f\left(y_{1,1}\right)-\frac{K_{2}}{\Delta x^{2}} y_{1,2}\right] \Rightarrow \\
\alpha_{N}= & \frac{1}{\left(K_{1} / \Delta x^{2}\right)}\left[\dot{\alpha}_{N-1}-k_{p, N}\left(y_{1,1}-\alpha_{N-1}\right)\right. \\
& \left.+\frac{2 K_{1}}{\Delta x^{2}} y_{1,1}-f\left(y_{1,1}\right)-\frac{K_{2}}{\Delta x^{2}} y_{1,2}\right]
\end{aligned}
$$

Consequently, the computation of the control input $\alpha_{N}=\rho_{0}$ which is exerted on the PDE model is performed backwards, by substituting recursively into $\rho_{0}$ the virtual control inputs $a_{N-1}, a_{N-2}, \ldots, a_{i}, \ldots, a_{2}, a_{1}$.

\section{Closed Loop Dynamics}

By substituting Eq. (42) into Eq. (35) of the state-space model of the PDE dynamics, and using the definition $y_{1,1}-y_{1,1}^{d}=$ $z_{1}$ one has

$$
\begin{aligned}
\dot{y}_{1,1} & =\dot{y}_{1,1}^{d}-k_{p, 1}\left(y_{1,1}-y_{1,1}^{d}\right) \\
& \Rightarrow\left(\dot{y}_{1,1}-\dot{y}_{1,1}^{d}\right)+k_{p, 1}\left(y_{1,1}-y_{1,1}^{d}\right)=0 \\
& \Rightarrow \dot{z}_{1}+k_{p, 1} z_{1}=0
\end{aligned}
$$

Equivalently, by substituting Eqs. (41) into (34), and using the definition $y_{1,2}-\alpha_{1}=z_{2}$ one has

$$
\begin{aligned}
\dot{y}_{1,2} & =\dot{\alpha}_{1}-k_{p, 2}\left(y_{1,2}-\alpha_{1}\right) \\
& \Rightarrow\left(\dot{y}_{1,2}-\dot{\alpha}_{1}\right)+k_{p, 2}\left(y_{1,2}-\alpha_{1}\right)=0 \\
& \Rightarrow \dot{z}_{2}+k_{p, 2} z_{2}=0
\end{aligned}
$$

Similarly, continuing with the rest of the equations of the state-space model and by substituting Eq. (40) into Eq. (33), 
while also using the definition $y_{1,3}-\alpha_{2}=z_{3}$ one has

$$
\begin{aligned}
\dot{y}_{1,3} & =\dot{\alpha}_{2}-k_{p, 3}\left(y_{1,3}-\alpha_{2}\right) \\
& \Rightarrow\left(\dot{y}_{1,3}-\dot{\alpha}_{2}\right)+k_{p, 3}\left(y_{1,3}-\alpha_{2}\right)=0 \\
& \Rightarrow \ddot{z}_{3}+k_{p, 3} z_{3}=0
\end{aligned}
$$

Moving backwards, and by substituting Eqs. (39) into (32) of the state-space model of the PDE dynamics, and using the definition $y_{1, i}-\alpha_{i-1}=z_{i}$ one has

$$
\begin{aligned}
\dot{y}_{1, i} & =\dot{\alpha}_{i-1}-k_{p, i}\left(y_{1, i}-\alpha_{i-1}\right) \\
& \Rightarrow\left(\dot{y}_{1, i}-\dot{\alpha}_{i-1}\right)+k_{p, i}\left(y_{1, i}-\alpha_{i-1}\right)=0 \\
& \Rightarrow \dot{z}_{i}+k_{p, i} z_{i}=0
\end{aligned}
$$

By substituting Eqs. (37) into (30) of the state-space model of the PDE dynamics, and using the definition $y_{1, N-1}-\alpha_{N-2}=$ $z_{N-1}$ one has

$$
\begin{aligned}
\dot{y}_{1, N-1} & =\dot{\alpha}_{N-2}-k_{p, N-1}\left(y_{1, N-1}-\alpha_{N-2}\right) \\
& \Rightarrow\left(\dot{y}_{1, N-1}-\dot{\alpha}_{N-2}\right)+k_{p, N-1}\left(y_{1, N-1}-\alpha_{N-2}\right)=0 \\
& \Rightarrow \dot{z}_{N-1}+k_{p, N-1} z_{N-1}=0
\end{aligned}
$$

Finally, by substituting Eqs. (36) into (30), and using the definition $y_{1, N}-\alpha_{N-1}=z_{N}$ one obtains

$$
\begin{aligned}
\dot{y}_{1, N} & =\dot{\alpha}_{N-1}-k_{p, N}\left(y_{1, N}-\alpha_{N-1}\right) \\
& \Rightarrow\left(\dot{y}_{1, N}-\dot{\alpha}_{N-1}\right)+k_{p, N}\left(y_{1, N}-\alpha_{N-1}\right)=0 \\
& \Rightarrow \dot{z}_{N}+k_{p, N} z_{N}=0
\end{aligned}
$$

Thus, the dynamics of the closed-loop system becomes

$$
\begin{aligned}
& \dot{z}_{1}+k_{p, 1} z_{1}=0 \\
& \dot{z}_{2}+k_{p, 2 z_{2}}=0 \\
& \dot{z}_{3}+k_{p, 3} z_{3}=0 \\
& \ldots \ldots \\
& \dot{z}_{i}+k_{p, i} z_{i}=0 \\
& \ldots \ldots \\
& \dot{z}_{N-1}+k_{p, N-1} z_{N-1}=0 \\
& \dot{z}_{N}+k_{p, N} z_{N}=0
\end{aligned}
$$

The dynamics of the closed-loop system can be also written in matrix form

$$
\ddot{\tilde{Z}}+K_{P} \tilde{Z}=0
$$

where $\tilde{Z}=\left[z_{1}, z_{2}, z_{3}, \ldots, z_{i}, \ldots, z_{N-1}, z_{N}\right]$, and $K_{P}=$ $\operatorname{diag}\left[k_{p, 1}, k_{p, 2}, k_{p, 3}, \ldots, k_{p, i}, \ldots, k_{p, N-1}, k_{p, N}\right]$.

After suitable selection of the coefficients $k_{P, i}, i=1$, $2, \ldots, N$ such that the monomials $p(s)=s+k_{p, i}$ to have a negative root, it can be assured that $\lim _{t \rightarrow \infty} z_{i}(t)=0$ and that the closed-loop system is asymptotically stable.

Moreover, to prove asymptotic stability for the closed-loop system, the following Lyapunov function can be used

$V_{L}=\sum_{i=1}^{N} \frac{1}{2} z_{i}^{2}$

The derivative of this Lyapunov function with respect to time is given by

$$
\begin{aligned}
\dot{V}_{L} & =\sum_{i=1}^{N} z_{i} \dot{z}_{i} \Rightarrow \dot{V}_{L}=\sum_{i=1}^{N} z_{i}\left(-k_{p_{i}} z_{i}\right) \\
& \Rightarrow \dot{V}_{L}=-\sum_{i=1}^{N} k_{p, i} z_{i}^{2}<0
\end{aligned}
$$

Thus, it is proven again that the closed-loop system is globally asymptotically stable.

\section{Simulation Tests}

Simulation examples about the proposed control method for distributed parameter systems are provided for the case of the Fokker-Planck PDE that was given in Eq. (10). The obtained results are depicted in Figs. 1, 2, 3, 4 and 5. The spatial discretization of the PDE model consisted of $N=25$ points. The sampling period was chosen to be $T_{S}=0.01 \mathrm{sec}$. A normalized scale has been used for the $\rho(x, t)$ axis. The boundary condition $\rho_{N+1}$ of the PDE was taken to be known and constant. The boundary condition $\rho_{0}$ served as the control input and was computed as each iteration of the control algorithm according to the procedure described in "Computation of a Boundary Conditions-Based Feedback Control Law" section. "The value of $\rho(x, t)$ " on the vertical axis of the plot appearing in Fig. 1 has been normalized.

The numerical simulation experiments have confirmed the theoretical findings of this article. It has been shown that by

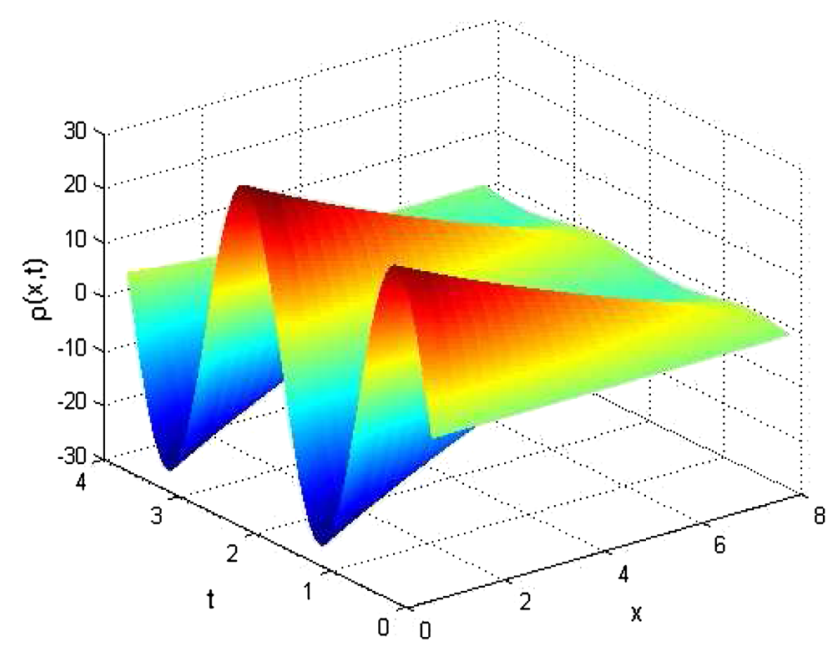

Fig. 1 Fokker-Planck PDE under boundary feedback control 
Fig. 2 a tracking of reference setpoint by the value of the FP PDE at the final grid point $x_{N} \mathbf{b}$ control input

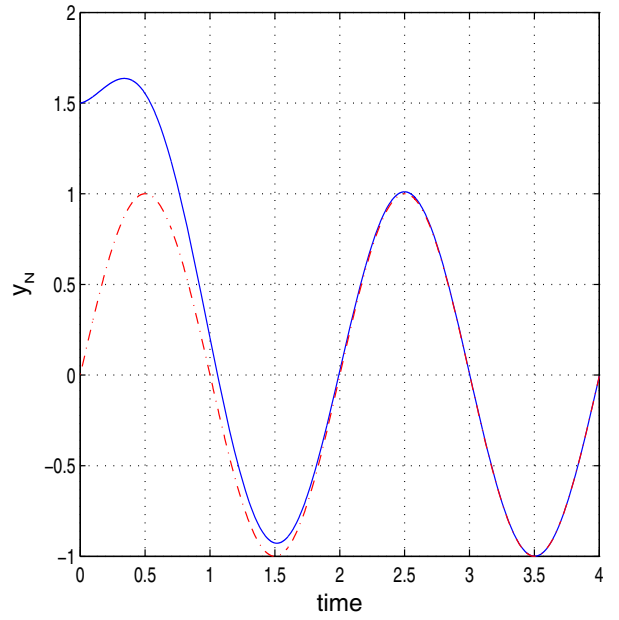

(a)
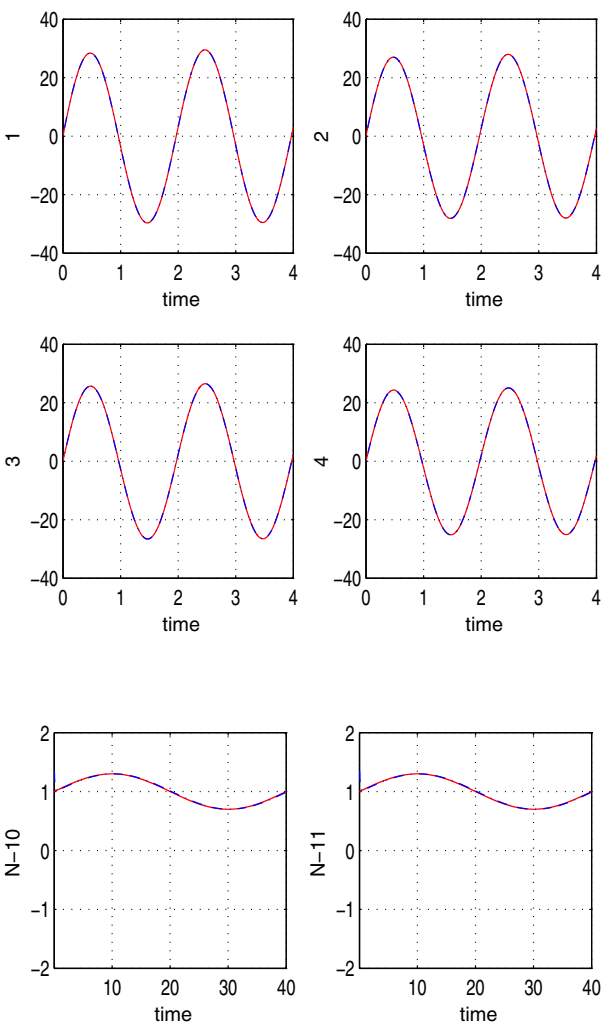

Fig. 4 a variation in time of the solution of the PDE at grid points $x_{9}$ to $x_{12}$ (blue line) and associated reference setpoint (red line) $\mathbf{b}$ variation in time of the solution of the PDE at grid points $x_{13}$ to $x_{16}$ (blue line) and associated reference setpoint (red line)

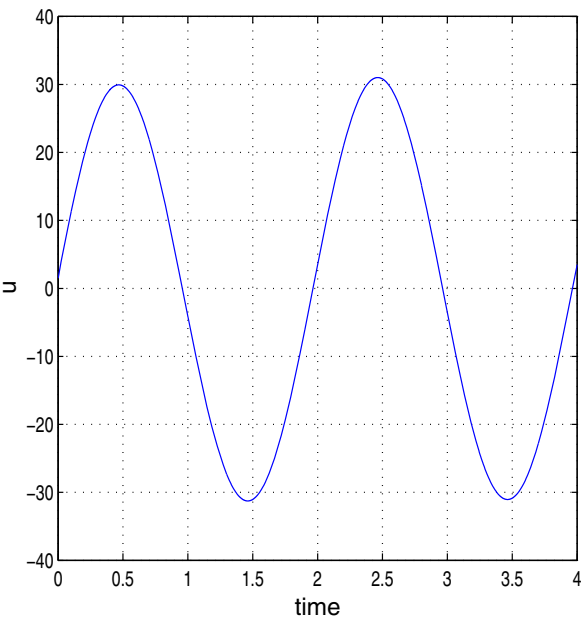

(b)
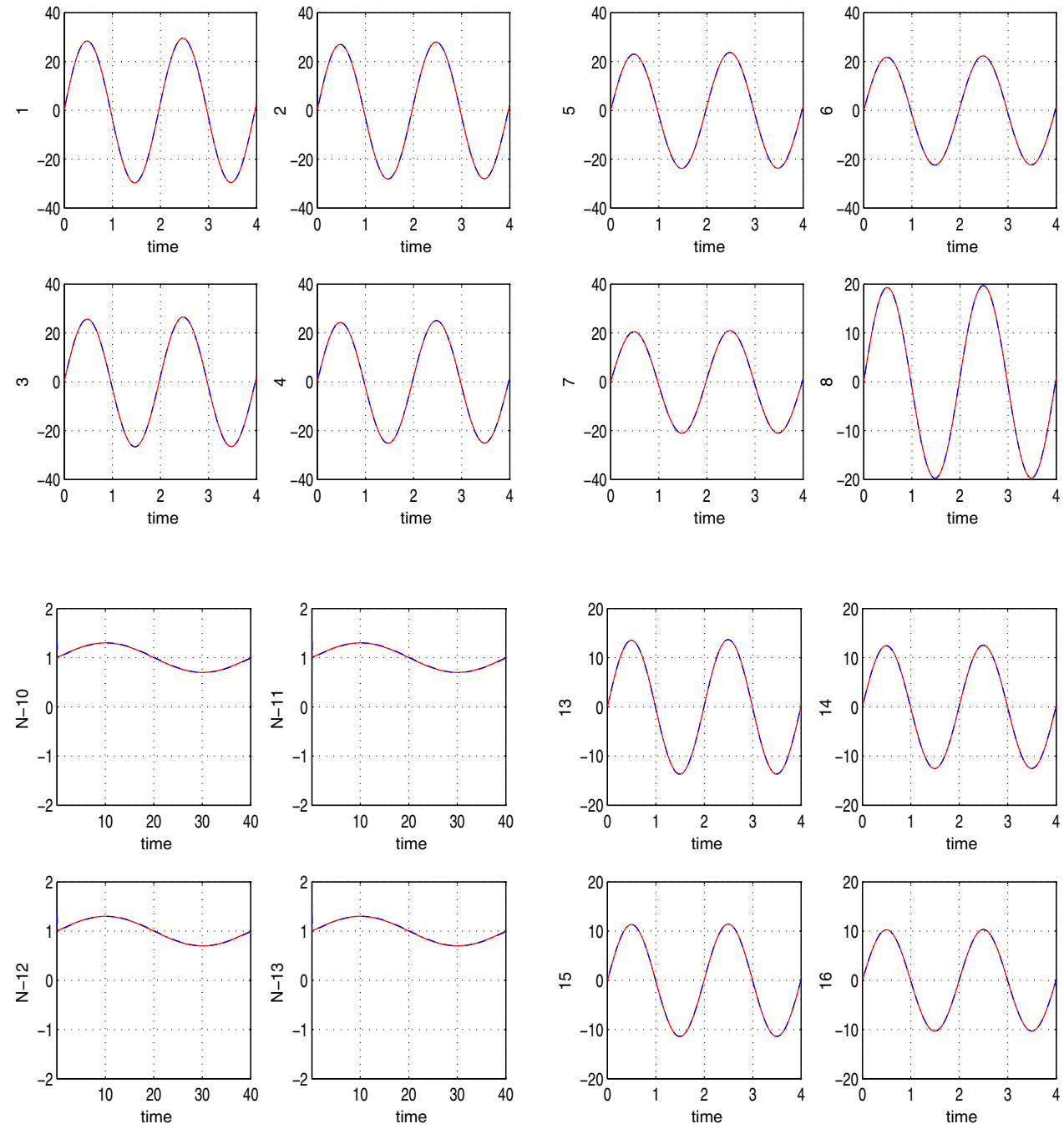

(a)
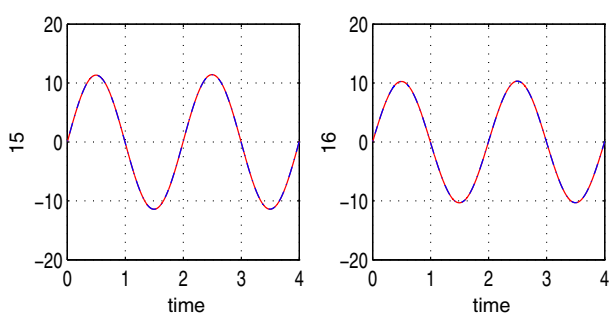

(b) 
Fig. 5 a variation in time of the solution of the PDE at grid points $x_{17}$ to $x_{20}$ (blue line) and associated reference setpoint (red line) $\mathbf{b}$ variation in time of the solution of the PDE at grid points $x_{21}$ to $x_{24}$ (blue line) and associated reference setpoint (red line)
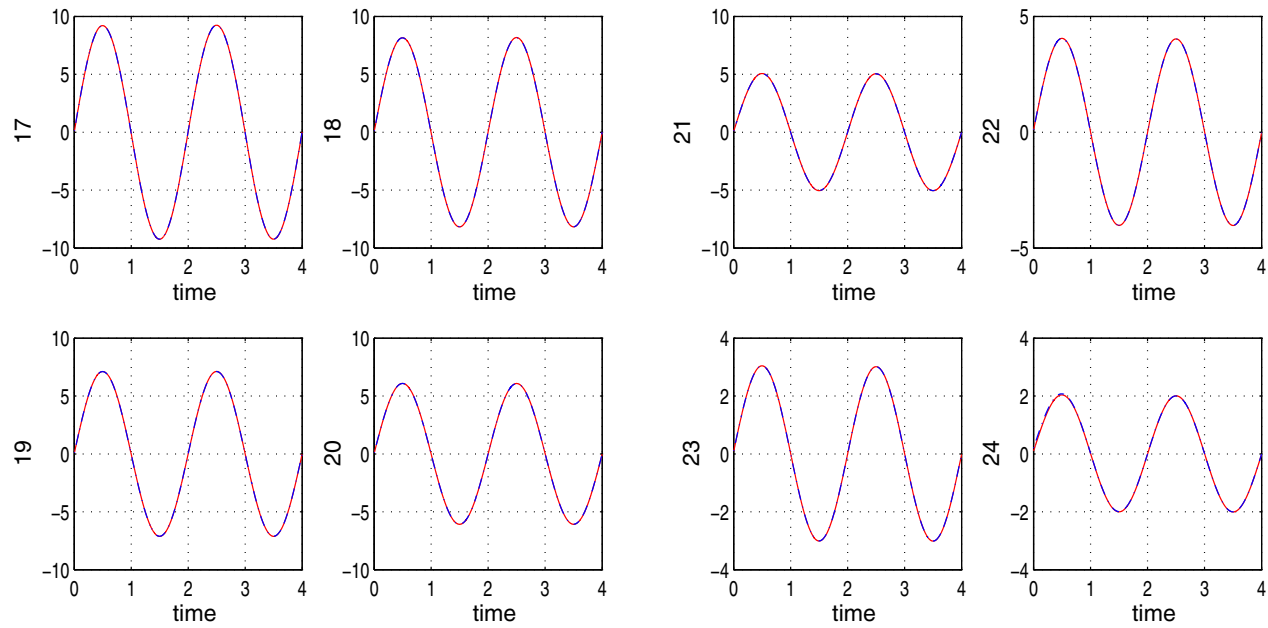

(a)
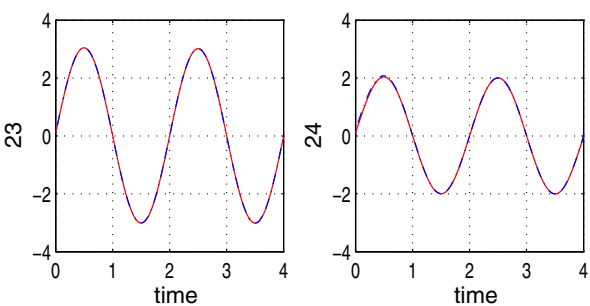

(b) applying the proposed control method, the Fokker-Planck PDE dynamics can be modified so as to converge to the desirable reference profile. The control input that achieves this changes smoothly and has a moderate range of variation. The accuracy of tracking of the reference setpoints was quite satisfactory. It is noted that the application of the proposed Fokker-Planck PDE feedback control method is also applicable to other diffusion phenomena.

\section{Conclusions}

A feedback control method of the Fokker-Planck PDE has been introduced, aiming at developing an approach for the stabilization of the associated diffusion process. Following semi-discretization and a finite differences scheme and the approach for numerical solution of partial differential equations, the Fokker-Planck PDE model has been decomposed into an equivalent set of nonlinear ODEs and a state-space model has been obtained. Next, it has been proven that each one of the aforementioned ODEs stands for a differentially flat subsystem. This enables to compute for each ODE subsystem a virtual control input which linearizes its dynamics and eliminates the associated output's tracking error. From the state equations that constitute the last subsystem one can find the boundary condition that also stands for the control input to the Fokker-Planck PDE model.

To compute the boundary control input of the Fokker-Planck PDE model one has to use recursively all virtual control inputs which are applied to the previously mentioned ODE subsystems. The control input of a subsystem incorporates the control input of its preceding subsystem. Thus, the computation of control inputs moves progressively from the last ODE system to the first one. Consequently, by tracing the rows of the state-space model backwards, at each iteration of the control algorithm, one can finally obtain the control input that should be applied to the Fokker-Planck PDE so as to assure that all its state vector elements will converge to the desirable setpoints. By analyzing the dynamics of the closed loop system that results from the application of the aforementioned control method, asymptotic stability is confirmed. Finally numerical simulation experiments have been provided about the application of the proposed control method to the model of the Fokker-Planck PDE. These performance tests have shown the control method's accuracy and reliability.

\section{References}

1. Rigatos, G.: Advanced Models of Neural Networks: Nonlinear Dynamics and Stochasticity in Biological Neurons. Springer, New York (2013)

2. Jedrzejewski, F.: Modèles Aléatoires et Physique Probabiliste. Springer, New York (2009)

3. Klebaner, F.: Introduction to Stochastic Calculus with Applications. Imperial College Press, London (2005)

4. Comets, F., Meyre, T.: Calcul Stochastique et Modèles de Diffusions. Dunod, Paris (2006)

5. Gitterman, M.: The Noisy Oscillator. World Scientific, Hackensack (2005)

6. Basseville, M., Nikiforov, I.: Detection of Abrupt Changes: Theory and Applications. Prentice Hall, Englewood Cliffs (1993)

7. Faris, W.: Diffusion, Quantum Theory and Radically Elementary Mathematics. Princeton University Press, Princeton (2006)

8. Faccioli, P., Sega, M., Pelerino, F., Orland, H.: Dominant pathways in protein folding. Physical Review Letters 97, 108101 (2006)

9. Carlier, G., Salomon, J.: A monotonic algorithm for the optimal control of the Fokker-Planck equation, American Control Conference (2010)

10. Cáceres, M., Carrillo, J., Tao, L.: A numerical solver for a nonlinear Fokker-Planck equation representation of neuronal network dynamics. J. Comput. Phys. 230, 1084-1099 (2011)

11. Sjoberg, P., Lotstedt, P., Elf, J.: Fokker-Planck approximation of the master equation in molecular biology. Comput. Vis. Sci. 12, $37-50$ (2009) 
12. Olivier, F., Sedoglavic, A.: A generalization of flatness to nonlinear systems of partial differential equations: Application to the control of a flexible rod. In: Proceedings of the 5th IFAC symposium on nonlinear control systems, Saint-Petersbourg (2001)

13. Kröner, A.: Adaptive finite element methods for optimal control second order hyberbolic equations. Comput. Methods Appl. Math. 11(2), 214-240 (2011)

14. Guo, L., Billings, S.A.: State-space reconstruction and spatiotemporal prediction of lattice dynamical systems. IEEE Trans. Autom. Control 52(4), 622-632 (2007)

15. Pinsky, M.: Partial differential equations and boundary value problems. Prentice-Hall, Upper Saddle River (1991)

16. Utz, T., Meurer, T., Kugi, A.: Trajectory planning for twodimensional quasi-linear parabolic PDE based on finite diffference semi-discretization, 18th IFAC World Congress. Milano (2011)

17. Balogh, A., Kristic, M.: Infinite dimensional backstepping style feedback transformations for a heat equation with an arbitrary level of instability. Eur. J. Control 8, 165-175 (2002)

18. Smyshlyaev, A., Krstic, M.: Adaptive Control of Parabolic PDEs. Princeton University Press, Princeton (2010)

19. Boskovic, D.M., Krstic, M., Liu, W.J.: Boundary control of an unstable heat equation via measurement of domain averaged temperature. IEEE Trans. Autom. Control 46, 2022-2028 (2002)

20. Liu, W.J.: Boundary stabilization of an unstable heat equation. SIAM J. Control Optim. 42, 1033-1043 (2003)

21. Maidi, A., Corriou, J.P.: Distributed control of nonlinear diffusion systems by input-output linearization. Int. J. Robust and Nonlinear Control 26, 389-405 (2014)

22. Zwart, H., Le Gorrec, Y., Maschke, B.: Linking hyperbolic and parabolic PDEs, 2011 50th IEEE conference on decision and control and european control conference, CDC-ECC, Orlando (2011)

23. Woitteneck, F., Mounier, H.: Controllability of networks of spatially one-dimensional second order PDEs-an algebraic approach. SIAM J. Control Optim. 48(6), 3882-3902 (2010)

24. Mounier, H., Rudolph, J., Wouttenneck, F.: Boundary Value Problems and Convolutional Systems Over Rings of Ultradistributions, Advances in the Theory of Control, Signal and Systems with Physical Modelling. Lecture notes in control an information sciences, pp. 179-188. Springer, New York (2010)
25. Fliess, M., Mounier, H.: An algebraic framework for infinitedimensional linear systems, In Proceedings of of international school on automatic control of lille, control of distributed parameter systems: Theory and applications, Grenoble (2002)

26. Winkler, F.J., Lohmann, B.: Design of a decoupling controller structure for first order hyperbolic PDEs with distributed control action, 2010 American Control Conference. Baltimore (2010)

27. Laroche, B., Martin, P., Rouchon, P.: Motion planning of the heat equation. Int. J. Robust Nonlinear Control 40(8), 629-643 (2000)

28. Bensoussan, A., Prato, G.D., Delfour, M.C., Mitter, S.K.: Representation and control of infinite dimensional systems. Birkahaüser, Boston (2006)

29. I. Boussaada, A. Cela, H. Mounier and S.I. Niculescu, Control of Drilling Vibrations: A time-delay system-based approach, 11th Workshop on Time Delay Systems (2013)

30. Winkler, F., Krause, I., Lohmann, B.: Flatness-based control of a continuous furnace, 18th Intl. Conference on Control Applications, Part of 2009 IEEE Multi-Conference on Systems and Control. Saint Petersburg (2009)

31. Bououden, S., Boutat, D., Zheng, G., Barbot, J.P., Kratz, F.: A triangular canonical form for a class of 0-flat nonlinear systems. Int. J. Control 84(2), 261-269 (2011)

32. Lévine, J.: Analysis and Control of Nonlinear Systems: a flatnessbased approach. Springer, New York (2009)

33. Lévine, J.: On necessary and sufficient conditions for differential flatness. Appl. Algebra Eng. Commun. Comput. 22(1), 47-90 (2011)

34. Rudolph, J.: Flatness Based Control of Distributed Parameter Systems. Aachen, Examples and Computer Exercises from Various Technological Domains. Shaker, Aachen (2003)

35. Rigatos, G.: Nonlinear control and filtering using differential flatness approaches: applications to electromechanicsl systems. Springer, New York (2015)

36. Sira-Ramirez, H., Agrawal, S.: Differentially Flat Systems. Marcel Dekker, New York (2004) 